SUBJECT AREAS: PARKINSON'S DISEASE NEUROTRANSMITTERS

Received 21 August 2013

Accepted 20 December 2013

Published 16 January 2014

Correspondence and requests for materials should be addressed to W.G.M. (wassilios. meissner@chubordeaux.fr)

\section{L-dopa-induced dyskinesia: beyond an excessive dopamine tone in the striatum}

\author{
Gregory Porras ${ }^{1,2,3}$, Philippe De Deurwaerdere ${ }^{1,2}$, Qin Liं ${ }^{3,4}$, Matteo Marti $^{5}$, Rudolf Morgenstern ${ }^{6}$, \\ Reinhard Sohr ${ }^{6}$, Erwan Bezard ${ }^{1,2,3,4,7}$, Michele Morari ${ }^{8}$ \& Wassilios G. Meissner ${ }^{1,2,7,9}$
}

L-dopa remains the mainstay treatment for Parkinson's disease (PD), although in later stages, treatment is complicated by L-dopa-induced dyskinesias (LID). Current evidence links LID to excessive striatal L-dopa-derived dopamine (DA) release, while the possibility of a direct involvement of L-dopa itself in LID has been largely ignored. Here we show that L-dopa can alter basal ganglia activity and produce LID without enhancing striatal DA release in parkinsonian non-human primates. These data may have therapeutic implications for the management of advanced PD since they suggest that LID could result from diverse mechanisms of action of L-dopa.

\section{A}

lthough dyskinesias develop after several years of treatment, L-dopa remains the mainstay treatment for Parkinson's disease (PD). L-dopa-induced dyskinesias (LID) have consistently been related to excessive dopamine (DA) release ${ }^{1}$, leading to aberrant striatofugal neuron activity and hence over-reduced activity in the pallido-thalamic GABAergic pathway ${ }^{2,3}$. While there is a direct relationship between L-dopa dose and LID occurrence and severity, PET studies suggest that LID are not strictly paralleled by a raise in striatal DA tone $e^{4}$. Earlier findings have demonstrated that L-dopa may act on its own ${ }^{5}$. L-dopa produces some pharmacological actions not through conversion to dopamine ${ }^{6-8}$, which result into antiparkinsonian action of L-dopa itself unraveled in both the 6-hydroxydopamine-lesioned and reserpine-treated rat models of $\mathrm{PD}^{8,9}$. Such antiparkinsonian action happened (i) before the elevation in DA when L-dopa was administered after pretreatment with benserazide or (ii) in absence of DA when L-dopa was given in combination with NSD-1015, a central aromatic Lamino acid dopa decarboxylase (AADC) inhibitor?.

A direct role for L-dopa upon LID, independently from DA, has however received little attention beyond the classic conversion into $\mathrm{DA}^{10,11}$ (but see ${ }^{12}$ ), thereby calling for an investigation in the gold standard experimental model of PD. In the present study we show that L-dopa can alter basal ganglia activity and produce LID without enhancing striatal DA release in dyskinetic parkinsonian macaques.

\section{Results}

Systemic administration of L-dopa + benserazide (BZD) in 8 MPTP-treated macaque, monkeys previously made dyskinetic, significantly increased plasma $(t=20 \min : P=0.002 ; t=60 \min : P=0.003 ; t=100$ min: $P=0.005$; $\mathrm{t}=140$ min: $\mathrm{P}=0.05 ; \mathrm{t}=180 \mathrm{~min}: \mathrm{P}=0.11, \mathrm{n}=4$, Fig. $1 \mathrm{~A})$ and striatal $\mathrm{L}$-dopa levels $\left(\mathrm{chi}^{2}=24.2, \mathrm{df}=9, \mathrm{P}=\right.$ $0.004, \mathrm{n}=8$, Fig. 1B). Since area under curves (AUCs) of striatal L-dopa levels were not different after oral $(\mathrm{n}=4)$ or intravenous $(\mathrm{n}=4)$ administration $(\mathrm{P}=0.42$, Fig. $1 \mathrm{~B}$, inset), data from the two groups were pooled for further analysis.

Administration of $\mathrm{L}$-dopa did not alter extracellular levels of $\mathrm{DA}\left(\mathrm{chi}^{2}=11.9, \mathrm{df}=9, \mathrm{P}=0.22\right)$. This surprising result was first obtained in four monkeys receiving L-dopa $+\mathrm{BZD}$ orally $\left(\mathrm{chi}^{2}=8.3, \mathrm{df}=9, \mathrm{P}=0.50\right)$ and confirmed in four more animals after intravenous L-dopa $+\mathrm{BZD}\left(\mathrm{chi}^{2}=14.3, \mathrm{df}=9, \mathrm{P}=0.11\right)$ for excluding anesthesia-related reduced drug absorption. In the same animals, L-dopa + BZD administration significantly decreased extracellular levels of thalamic GABA from $112 \pm 36 \mathrm{nM}$ at baseline to $37 \pm 14 \mathrm{nM}$ at three hours after 


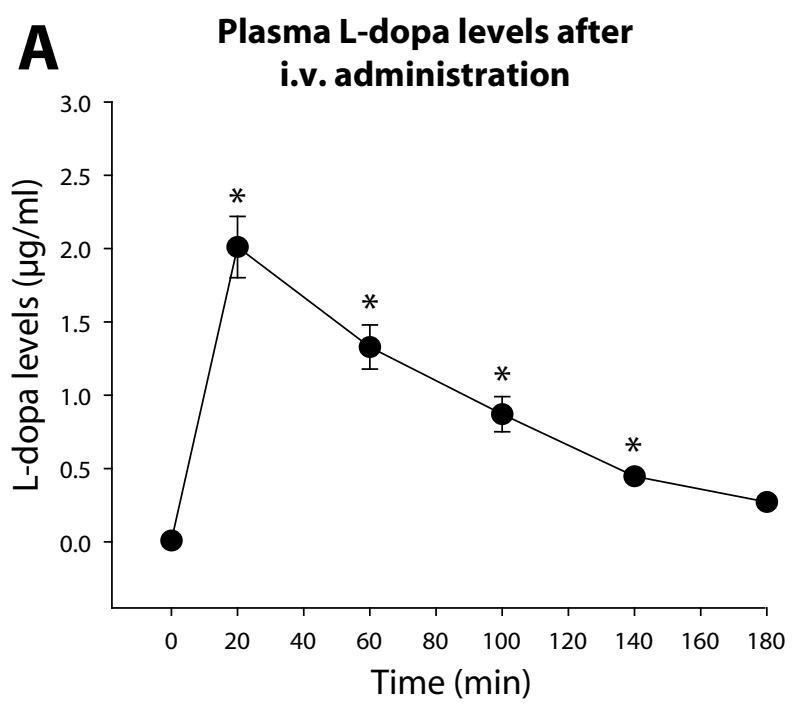

\section{B Striatal L-dopa and DA as well as thalamic GABA levels after systemic l-dopa}

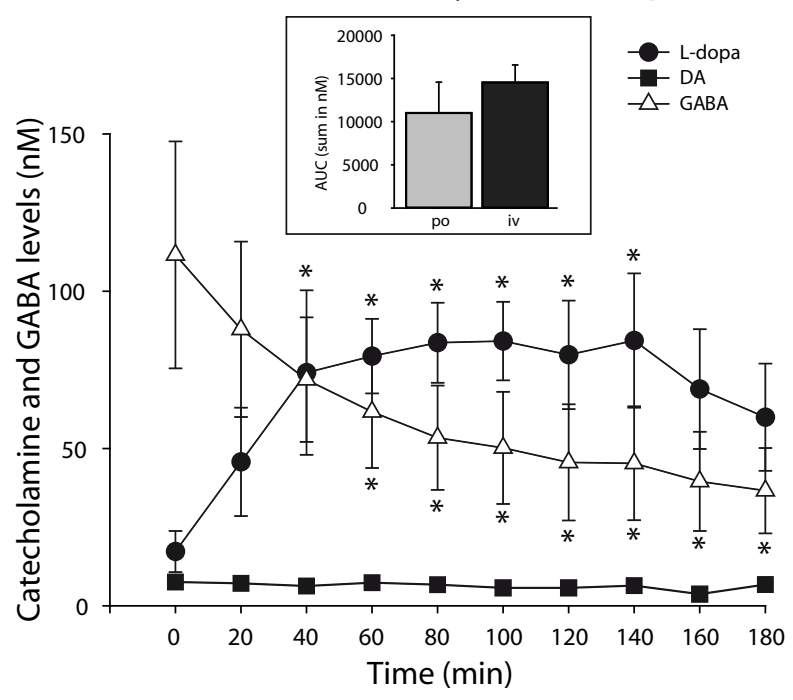

\section{Striatal DA and DOPAC levels during} reverse microdialysis in striatum

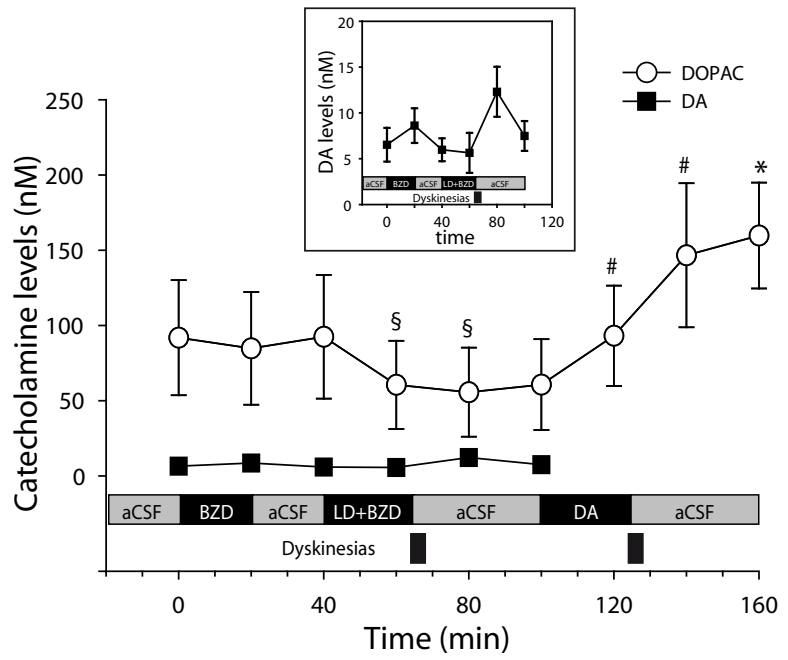

Figure 1 (A) L-dopa plasma levels after intravenous administration of $2.5 \mathrm{mg} / \mathrm{kg}$ together with benserazide $(\mathrm{n}=4)$. Peak L-dopa plasma concentrations correspond to morning L-dopa dosages in Parkinson's disease patients with advanced disease and motor fluctuations. Data are shown as mean concentration (in $\mathrm{nM}) \pm$ s.e.m. ${ }^{*} \mathrm{P}<0.05$ vs. baseline. (B) Time course of dialysate concentrations of DA and L-dopa in the striatum and GABA in the thalamus after L-dopa + BZD administration (time 0). Data correspond to the mean concentration \pm s.e.m. of the dialysate content expressed in $\mathrm{nM}$ and result from the combination of data from two modalities of L-dopa + BZD administration $(2.5 \mathrm{mg} / \mathrm{kg}$ i.v., $\mathrm{n}=4$ or $20 \mathrm{mg} / \mathrm{kg}$ p.o., $\mathrm{n}=4$ ). The inset reports the overall increase in extracellular L-dopa levels of each administration modality over the observation period of three hours. ${ }^{*} \mathrm{P}<0.05$ vs. baseline. (C) Time course of the striatal application of benserazide (BZD, $100 \mu \mathrm{M})$, L-dopa + BZD (100 $\mu \mathrm{M}$ each) or DA $(10 \mu \mathrm{M})$ on DA and DOPAC extracellular levels. Drugs were applied using reverse microdialysis. The period of infusion, initially scheduled to last 40 minutes, was stopped after the first signs of dyskinesia, in order to avoid persistent and long term dyskinesia (dyskinesia duration corresponds to black bars). DA was subsequently applied after a recovery period using the same criteria. The inset provides a magnification of extracellular DA concentrations. Dialysate contents are expressed as mean concentration (in $\mathrm{nM}) \pm$ s.e.m $(\mathrm{n}=2) .{ }^{\mathrm{s}} \mathrm{P}=0.09$ and $0.06 \mathrm{vs} \mathrm{t}=20 \mathrm{~min}$. ${ }^{*} \mathrm{P}=0.09$ and 0.06 vs $\mathrm{t}=100$ min. ${ }^{*} \mathrm{P}<0.05$ vs. $\mathrm{t}=100 \mathrm{~min} . \mathrm{aCSF}=$ artificial cerebrospinal fluid.

drug administration $\left(\mathrm{chi}^{2}=36.8 \mathrm{df}=9, \mathrm{P}<0.001\right.$, Fig. $\left.1 \mathrm{~B}\right)$, an unbiased index of the antiparkinsonian/prodyskinetic impact of the pharmacological treatment ${ }^{3,13,14}$.

In two awake parkinsonian macaques in which motor behavior was concomitantly blindly rated by trained observers, reverse dialysis of BZD alone $(100 \mu \mathrm{M})$ in striatum did not induce LID and did not modify striatal levels of DA $(\mathrm{P}=0.23$, Fig. $1 \mathrm{C})$ or DOPAC $(\mathrm{P}=$ $0.19)$. In contrast, equimolar concentrations of L-dopa $+\mathrm{BZD}$ (100 $\mu \mathrm{M}$ each) induced LID in the two awake MPTP-treated monkeys (Fig. 1C). The effect on behavior was paralleled by a trend for a decrease in extracellular levels of the direct DA metabolite DOPAC ( $t$ $=60$ min: $\mathrm{P}=0.087 ; \mathrm{t}=80 \min : \mathrm{P}=0.061)$. A trend for increased extracellular levels of DA was observed but without reaching significance $(t=60 \min : P=0.20 ; t=80$ min: $P=0.18$, Fig. $1 C$, inset $)$. According to observed effect size and standard deviation, the assessment of thirteen hemispheres would have been needed for reaching the threshold of significance with a power of $80 \%$. No significant changes were observed for 3-methoxytyramine (3-MT) $(\mathrm{t}=$ 60 min: $\mathrm{P}=1.0 ; \mathrm{t}=80$ min: $\mathrm{P}=0.37)$, the second direct metabolite of DA. Striatal infusion of DA $(10 \mu \mathrm{M})$ also induced LID (Fig. 1C). The effect was paralleled by a trend for increased extracellular levels of DOPAC that became significant at the last sample $(t=120 \mathrm{~min}: \mathrm{P}$ $=0.087 ; \mathrm{t}=140$ min: $\mathrm{P}=0.055 ; \mathrm{t}=160 \mathrm{~min}: \mathrm{P}=0.022 ;$ Fig. $1 \mathrm{C})$ as expected from the literature. Simultaneously, an increase of 3-MT levels was observed $(\mathrm{t}=120 \min : \mathrm{P}=0.19 ; \mathrm{t}=140 \min : \mathrm{P}=0.042 ; \mathrm{t}$ $=160$ min: $\mathrm{P}=0.030)$. This experiment demonstrates that both $\mathrm{L}$ dopa (without production of DA) and DA infusions lead to dyskinesia in awake monkeys suggesting different mechanisms of action for the same outcome.

\section{Discussion}

The present data demonstrate that L-dopa can elicit LID independently of a significant raise in striatal DA release, suggesting that LID could result from hitherto unexplored mechanisms of action of Ldopa. Since the two awake animals presented peak-dose dyskinesia, i.e. the condition where parkinsonian motor signs are best attenuated, the data support the concept that L-dopa may have antiparkinsonian effects independent of DA, at least in very advanced disease where DA levels are extremely low if existent ${ }^{8,9}$.

Puzzlingly, L-dopa did not enhance extracellular DA levels in the striatum of MPTP-treated monkeys bearing an almost complete lesion of nigro-striatal DA neurons ${ }^{15,16}$. It is unlikely that anesthesia had suppressed the ability of L-dopa to enhance DA release as L-dopa 
dose-dependently enhances striatal DA release in rats, whatever the dose or the nature of the anesthetic ${ }^{17,18}$. The use of two different routes of administration for L-dopa excludes pharmacokinetic concerns that may apply when using a single modality of administration. This is substantiated by increased extracellular L-dopa levels in the striatum whatever the modality of administration (inset Fig. 1B). Finally, peak L-dopa plasma concentrations measured here correspond to morning L-dopa dosages in PD patients with advanced disease and motor fluctuations ${ }^{19,20}$.

L-dopa had functional effects within the motor loop. Indeed, Ldopa administration decreased extracellular levels of GABA in the thalamus, an in vivo index of the activity of the pallido-thalamic GABAergic pathway, ${ }^{3,13,14}$. A strong decrease in the activity of GABAergic neurons in the internal pallidum is considered as a marker of hyperkinetic movements ${ }^{2}$, and this is compatible with severe LID presented by all animals before the experiment.

The conversion of L-dopa to DA in catecholamine neurons requires AADC. Striatal AADC protein levels are decreased by $70 \%$ in 6-hydroxydopamine (6-OHDA)-lesioned rats ${ }^{21}$. In MPTPtreated squirrel monkeys, the number of AADC immunoreactive fibers and terminals in the putamen is reduced by $36 \%$ and AADC activity by $64 \%{ }^{22,23}$. Chronic administration of L-dopa increases striatal AADC levels in 6-OHDA-lesioned rats to $100-120 \%$ of naïve animals, irrespective of their dyskinetic profile, while tyrosine hydroxylase $(\mathrm{TH})$ levels are unchanged ${ }^{21}$. By contrast, putaminal expression of AADC and TH are not modified by chronic L-dopa treatment in MPTP-treated squirrel monkeys ${ }^{23}$. These observations may explain in part the discrepancy between the excessive L-dopainduced increase of extracellular striatal DA in 6-OHDA rats ${ }^{18}$ and the lack of a significant effect in MPTP-treated non-human primates in the present study. In PD patients, putaminal AADC activity is decreased to $10 \%$ of control values, while L-dopa treatment seems not to have a significant effect on AADC activity as suggested by the results of a post-mortem study ${ }^{24}$.

Although we did not evaluate the integrity of serotonergic (5-HT) neurons in the present study, the main neurochemical system involved in L-dopa-induced DA release in advanced $\mathrm{PD}^{17,25,26}$, the lack of a DA response following L-dopa administration is consistent with low 5-HT activity imposed by our experimental conditions. Indeed, MPTP treatment in non-human primates reduces 5-HT levels in various brain regions ${ }^{27}$, while chronic L-dopa administration in rodents and monkeys alters 5-HT neuron function and reduces L-dopa-induced DA release ${ }^{28,29}$. The remaining dyskinesia after removing the serotonergic innervation in L-dopa-treated parkinsonian rats $^{26}$, a situation in which no L-dopa-induced DA release is observed ${ }^{17}$, is also in line with our study while the primate model might be more sensitive to this intriguing mechanism.

Our data in primates suggest the existence of another mechanism of LID. Indeed, in awake MPTP-treated non-human primates, dyskinetic movements were elicited by striatal application of both DA and L-dopa with BZD, the latter in the absence of any changes in DA release (Fig. 1C). It remains to be determined whether the effect of Ldopa involves a specific action on one striatal target or intracellular effects affecting the transmission of a neurotransmitter system. Earlier reports have shown in rat models of PD that L-dopa itself can mediate an antiparkinsonian action ${ }^{8,9}$. Interestingly such Ldopa-mediated antiparkinsonian effects were significantly reduced by the alpha $(2 \mathrm{C})$ antagonist rauwolscine and the 5-HT(2C) agonist MK212 but not by the D2 dopamine receptor antagonist remoxipride or the D1 dopamine receptor antagonist SCH23390, suggesting that non-DA binding sites might be responsible for the generation of at least some L-dopa actions in this reserpine-treated rat $\operatorname{model}^{8}$. The pro-dyskinetic action of L-dopa was however not found in the 6OHDA rat model ${ }^{12}$ as it was solely attributed to its conversion into $\mathrm{DA}^{10,11}$. The opposite finding is however undisputedly shown in the present experimental setting in the MPTP-lesioned macaque. This discrepancy may account for the differential sensitivity of rodents versus primates to DA depletion, the more complex behavior of primates as well as the shared brain anatomy between macaques and humans which renders the present analysis more clinically relevant ${ }^{30,31}$.

Our data may have important implications for the management of advanced PD. Strategies aimed at lowering striatal DA tone such as 5$\mathrm{HT}_{1 \mathrm{~A}}$ receptor agonists that are currently being developed for the treatment of $\mathrm{LID}^{32}$ may be successful in PD as long as a sufficient number of 5-HT neurons is preserved. These strategies may not be pertinent in very advanced disease due to progressive loss of these neurons ${ }^{33}$ and where LID may thus more relate to direct effects of Ldopa than of DA. Other targets should therefore be studied including glutamatergic and adrenergic pathways to better understand the mechanisms of LID that occur independently from excessive DA release.

\section{Methods}

Animals. Nine macaca mulatta (5 males and 4 females, Xierxin, China) were housed under controlled conditions of humidity, temperature, and light (12-h light/12-h dark cycle, lights on at $8.00 \mathrm{am}$ ); food and water were available ad libitum. Experiments were carried out in accordance with European Communities Council Directive of 3 June 2010 (2010/6106/EU) for care of laboratory animals in an AAALAC-accredited facility following acceptance of study design by the Institute of Lab Animal Science Institutional Animal Care and Use Committee (Chinese Academy of Science, Beijing, China). Parkinsonism and dyskinesia were induced and rated as previously described $^{34,35}$ in accordance with the latest guidelines ${ }^{30}$. Briefly, macaques received daily i.v. injections of 1-methyl-4-phenyl-1,2,3,6-tetrahydropyridine (MPTP) hydrochloride $(0.2 \mathrm{mg} / \mathrm{kg})$ until they displayed parkinsonian symptoms. Once symptoms had stabilized, macaques were treated p.o. with Modopar (L-dopa/ benserazide, ratio $4: 1$ ) for 6 months at doses (15-20 mg/kg b.i.d.) individually adjusted to fully reverse parkinsonian symptoms. Both parkinsonian symptoms and dyskinesia severity were rated when animals were awake as previously described ${ }^{34-36}$. Median parkinsonian scores were $6.0 \pm 1.3$ in OFF condition and $0.0 \pm 0.3$ after Ldopa administration (the maximum disability score is 10 points indicating moderate to severe parkinsonism of the animals in the OFF condition). Median dyskinesia scores in $\mathrm{ON}$ condition were $3.0 \pm 0.5$ indicating marked dyskinesia interfering with normal activity. Our MPTP protocol leads to a reproducible near complete nigrostriatal lesion $(>95 \%)^{34,35}$.

Stereotactic surgery. Customized guide cannulae (CMA 12, Kista, Sweden) were placed above the rostro-caudal motor striatum and the ventro-lateral motor thalamus under stereotactic guidance using ventriculography and postoperative $\mathrm{x}$-ray while isoflurane anesthetized (unilaterally in animals for microdialysis under isoflurane anesthesia and bilaterally in animals where reverse microdialysis was performed while awake). Guide cannulae were permanently fixed to the skull with surgical screws and dental acrylic resin.

Microdialysis procedure. Immediately for anesthetized animals $(n=8)$ or one week after surgery for awake animals $(n=2)$, concentric dialysis probes (CMA 12, $4 \mathrm{~mm}$ length, $240 \mu \mathrm{m}$ outer diameter) were lowered through the guide cannula to reach the targets. Probes were perfused with artificial cerebrospinal fluid $(2 \mu \mathrm{l} / \mathrm{min}$, CMA, Kista, Sweden). After 120 minutes of stabilization, dialysates $(40 \mu \mathrm{l})$ were collected every $20 \mathrm{~min}$ in $10 \mu \mathrm{l}$ of $0.1 \mathrm{M}$ perchloric acid. Twenty microliters served for dosing catecholamines and $20 \mu \mathrm{l}$ (after neutralization with borate buffer) for gammaaminobutyric acid (GABA). Probe in vitro recoveries were around $10 \%$.

\section{High-performance liquid chromatography (HPLC). Blood samples were} centrifuged at $13,000 \mathrm{rpm}$ for $30 \mathrm{~min}$ at $4^{\circ} \mathrm{C}$. Aliquots $(50 \mu \mathrm{l})$ of the supernatants were stored at $-80^{\circ} \mathrm{C}$ until their use in biochemical assays. Before analysis by reversephase high performance liquid chromatography coupled with electrochemical detection $^{18}$, plasma samples were diluted $(1 / 8)$ with perchloric acid $(0.1 \mathrm{~N})$ and centrifuged, supernatants were injected into the HPLC system. Microdialysis samples for the assessment of L-dopa DA and its metabolites DOPAC and 3-MT were also stored at $-80^{\circ} \mathrm{C}$ until their use in biochemical assays. For monoamine measurements, a volume of $20 \mu \mathrm{l}$ was injected with an autosampler SIL-10AD vp (Shimadzu, Duisburg, Germany). Samples were separated on a HPLC column (ProntoSil $\mathrm{C} 18 \mathrm{SH}$ ) at a flow rate of $0.3 \mathrm{ml} / \mathrm{min}$. The mobile phase consisted of $80 \mathrm{mM}$ sodium dihydrogen phosphate, $44 \mathrm{mM}$ phosphoric acid, $0.5 \mathrm{mM}$ ethylenediaminetetraacetic acid (disodium salt), $0.85 \mathrm{mM} 1$-octanesulfonic acid (sodium salt), $2 \mathrm{mM}$ sodium chloride and 2.8\% 2-propanol, and was delivered with a LC10-AD vp pump (Shimadzu, Duisburg, Germany). Monoamines were quantified electrochemically by a glassy carbon electrode at a potential of $0.8 \mathrm{~V}(\mathrm{Ag} / \mathrm{AgCl}$ electrode). Sensitivity was set at 2-50 nA/V with an electrochemical detector (Antec Decade, Antec, Leyden, The Netherlands). The chromatograms were recorded with a chromatographic data system and quantified by determination of peak areas in relation to standard (Class vp, Shimadzu, Duisburg, Germany). 
GABA was determined by HPLC and fluorescence detection ${ }^{13}$. Samples were precolumn derivatized with o-phthalaldehyde-2-mercaptoethanol using an autoinjector and subsequently separated on a 5-C18 Chromsep analytical column (Chrompack, Middelburg, Netherlands) perfused at $0.48 \mathrm{ml} / \mathrm{min}$ with a mobile phase containing $0.1 \mathrm{M}$ sodium acetate, $10 \%$ methanol and $2.2 \%$ tetrahydrofuran ( $\mathrm{pH} 6.5$ ). GABA was detected by a FP-2020 Plus spectrophotometer (Jasco, Tokyo, Japan) with the excitation and the emission wavelengths set at 370 and $450 \mathrm{~nm}$, respectively.

Pharmacological treatment. Treatments were applied after baseline sampling. Four animals received $20 \mathrm{mg} / \mathrm{kg}$ Modopar p.o. (L-dopa/benserazide, BZD; $4: 1$ ratio), i.e. a dose that turned them ON when awake. To exclude reduced drug absorption because of anesthesia, four more animals received an i.v. injection with an equivalent dose (i.e. $2.5 \mathrm{mg} / \mathrm{kg}$ L-dopa methyl ester and $0.625 \mathrm{mg} / \mathrm{kg}$ BZD i.v.).

The equivalence dose of L-dopa dose for intravenous administration was determined in one non-human primate receiving on three consecutive days either $20 \mathrm{mg}$ / $\mathrm{kg}$ Modopar p.o. (L-dopa/benserazide, ratio $4: 1$ ), $2.5 \mathrm{mg} / \mathrm{kg}$ or $5 \mathrm{mg} / \mathrm{kg}$ levodopa methylester i.v. together with benserazide. Blood samples were centrifuged at $13,000 \mathrm{rpm}$ for $30 \mathrm{~min}$ at $4^{\circ} \mathrm{C}$. Before analysis with HPLC-ECD, plasma samples were diluted $(1 / 8)$ with perchloric acid $(0.1 \mathrm{~N})$ and centrifuged, supernatants were injected into the HPLC system. L-dopa plasma levels were assessed at baseline and after 15, 30, 60 and 90 minutes after L-dopa administration. Areas under the curve were 46488 for $20 \mathrm{mg} / \mathrm{kg}$ p.o., 54783 for $2.5 \mathrm{mg} . \mathrm{kg}$ i.v. and 92397 for $5 \mathrm{mg} / \mathrm{kg}$ i.v. Following these results, animals received $2.5 \mathrm{mg} / \mathrm{kg}$ i.v. during the microdialysis experiments.

BZD $(100 \mu \mathrm{M})$, L-dopa/BZD $(100 \mu \mathrm{M}$ each) or DA $(10 \mu \mathrm{M})$ were locally applied into the striatum by reverse dialysis in two awake animals that were seated in a primate chair to assess the potential of each drug to induce LID. To avoid long-lasting LID, drug infusion was stopped immediately after the onset of abnormal movements. All drugs except Modopar (Roche, Basel, Switzerland) were purchased from SigmaAldrich (France).

Statistical analysis. A repeated measure ANOVA on ranks was applied to estimate overall significance for levels of L-dopa, DA and GABA in anesthetized animals, followed by post hoc $t$-tests corrected for multiple comparisons versus baseline by the method of Dunnett. Striatal L-dopa levels (AUCs) after p.o. and i.v. treatment were compared by a $t$-test. Baseline L-dopa plasma concentrations were compared with levels at 20,60,100,140 and 180 minutes after intravenous L-dopa administration by using a paired $t$-test. For data obtained in two awake animals, a paired $t$-test or a signed rank test (if data were not normally distributed) were performed for assessing the effect of BZD $(t=0$ compared to $t=20)$, $\mathrm{L}$-dopa $+\mathrm{BZD}(\mathrm{t}=20$ compared to $\mathrm{t}=$ 60 and $t=80)$ and DA $(t=100$ compared to $t=120, t=140$ and $t=160)$. Data are presented as mean \pm standard error of the mean if not otherwise indicated. Statistical analysis was performed with Sigma Plot software (Version 12, Systat Software Inc., Chicago, IL, USA). Significance level was set at $\mathrm{P}<0.05$.

1. Carta, M. \& Bezard, E. Contribution of pre-synaptic mechanisms to L-DOPAinduced dyskinesia. Neuroscience 198, 245-251 (2011).

2. DeLong, M. R. Primate models of movement disorders of basal ganglia origin. Trends Neurosci. 13, 281-285 (1990).

3. Marti, M. et al. Nociceptin/orphanin FQ receptor agonists attenuate L-DOPAinduced dyskinesias. J Neurosci 32, 16106-16119 (2012).

4. de la Fuente-Fernandez, R. et al. Levodopa-induced changes in synaptic dopamine levels increase with progression of Parkinson's disease: implications for dyskinesias. Brain 127, 2747-2754 (2004).

5. Misu, Y., Goshima, Y., Ueda, H. \& Okamura, H. Neurobiology of L-DOPAergic systems. Prog.Neurobiol. 49, 415-454 (1996).

6. Goshima, Y., Kubo, T. \& Misu, Y. Biphasic actions of L-DOPA on the release of endogenous noradrenaline and dopamine from rat hypothalamic slices. $\mathrm{Br} \mathrm{J}$ Pharmacol 89, 229-234 (1986).

7. Misu, Y. \& Goshima, Y. Is L-dopa an endogenous neurotransmitter? Trends Pharmacol Sci 14, 119-123 (1993).

8. Alachkar, A., Brotchie, J. M. \& Jones, O. T. Locomotor response to L-DOPA in reserpine-treated rats following central inhibition of aromatic L-amino acid decarboxylase: further evidence for non-dopaminergic actions of L-DOPA and its metabolites. Neurosci Res 68, 44-50 (2010).

9. Nakazato, T. \& Akiyama, A. Effect of exogenous L-dopa on behavior in the rat: an in vivo voltammetric study. Brain Res 490, 332-338 (1989).

10. Buck, K. \& Ferger, B. Comparison of intrastriatal administration of noradrenaline and 1-DOPA on dyskinetic movements: a bilateral reverse in vivo microdialysis study in 6-hydroxydopamine-lesioned rats. Neuroscience 159, 16-20 (2009).

11. Buck, K., Voehringer, P. \& Ferger, B. Site-specific action of L-3,4dihydroxyphenylalanine in the striatum but not globus pallidus and substantia nigra pars reticulata evokes dyskinetic movements in chronic L-3,4dihydroxyphenylalanine-treated 6-hydroxydopamine-lesioned rats. Neuroscience 166, 355-358 (2010).

12. Buck, K. \& Ferger, B. Intrastriatal inhibition of aromatic amino acid decarboxylase prevents 1-DOPA-induced dyskinesia: a bilateral reverse in vivo microdialysis study in 6-hydroxydopamine lesioned rats. Neurobiol Dis 29, 210-220 (2008).

13. Marti, M., Trapella, C., Viaro, R. \& Morari, M. The nociceptin/orphanin FQ receptor antagonist J-113397 and L-DOPA additively attenuate experimental parkinsonism through overinhibition of the nigrothalamic pathway. J Neurosci 27, 1297-1307 (2007)

14. Stefani, A. et al. The clinical efficacy of L-DOPA and STN-DBS share a common marker: reduced GABA content in the motor thalamus. Cell Death Dis 2, e154 (2011).

15. Fernagut, P. O. et al. Dopamine transporter binding is unaffected by L-DOPA administration in normal and MPTP-treated monkeys. PLoS One 5, e14053 (2010).

16. Guigoni, C. et al. Levodopa-induced dyskinesia in MPTP-treated macaques is not dependent on the extent and pattern of nigrostrial lesioning. Eur J Neurosci 22, 283-287 (2005).

17. Navailles, S., Bioulac, B., Gross, C. \& De Deurwaerdere, P. Serotonergic neurons mediate ectopic release of dopamine induced by L-DOPA in a rat model of Parkinson's disease. Neurobiol Dis 38, 136-143 (2010).

18. Meissner, W. et al. Increased slow oscillatory activity in substantia nigra pars reticulata triggers abnormal involuntary movements in the 6-OHDA-lesioned rat in the presence of excessive extracellular striatal dopamine. Neurobiol.Dis. 22 586-598 (2006)

19. Ruottinen, H. M. \& Rinne, U. K. Entacapone prolongs levodopa response in a one month double blind study in parkinsonian patients with levodopa related fluctuations. J Neurol Neurosurg Psychiatry 60, 36-40 (1996).

20. Hauser, R. A. et al. Crossover comparison of IPX066 and a standard levodopa formulation in advanced Parkinson's disease. Mov Disord 26, 2246-2252 (2011)

21. Gil, S., Park, C., Lee, J. \& Koh, H. The roles of striatal serotonin and L -amino-acid decarboxylase on L-DOPA-induced Dyskinesia in a Hemiparkinsonian rat model. Cell Mol Neurobiol 30, 817-825 (2010).

22. Yee, R. E. et al. Nigrostriatal reduction of aromatic L-amino acid decarboxylase activity in MPTP-treated squirrel monkeys: in vivo and in vitro investigations. J Neurochem 74, 1147-1157 (2000).

23. Stephenson, D. T. et al. Differential loss of presynaptic dopaminergic markers in Parkinsonian monkeys. Cell Transplant 16, 229-244 (2007).

24. Lloyd, K. G., Davidson, L. \& Hornykiewicz, O. The neurochemistry of Parkinson's disease: effect of L-dopa therapy. J Pharmacol Exp Ther 195, 453-464 (1975).

25. Tanaka, H. et al. Role of serotonergic neurons in L-DOPA-derived extracellular dopamine in the striatum of 6-OHDA-lesioned rats. Neuroreport 10, 631-634 (1999).

26. Carta, M., Carlsson, T., Kirik, D. \& Bjorklund, A. Dopamine released from 5-HT terminals is the cause of L-DOPA-induced dyskinesia in parkinsonian rats. Brain 130, 1819-1833 (2007)

27. Pifl, C., Schingnitz, G. \& Hornykiewicz, O. Effect of 1-methyl-4-phenyl-1,2,3,6 tetrahydropyridine on the regional distribution of brain monoamines in the rhesus monkey. Neuroscience 44, 591-605 (1991).

28. Munoz, A. et al. Combined 5-HT1A and 5-HT1B receptor agonists for the treatment of L-DOPA-induced dyskinesia. Brain 131, 3380-3394 (2008).

29. Navailles, S., Bioulac, B., Gross, C. \& De Deurwaerdere, P. Chronic L-DOPA therapy alters central serotonergic function and L-DOPA-induced dopamine release in a region-dependent manner in a rat model of Parkinson's disease. Neurobiol Dis 41, 585-590 (2011).

30. Fox, S. H., Johnston, T. H., Li, Q., Brotchie, J. \& Bezard, E. A critique of available scales and presentation of the Non-Human Primate Dyskinesia Rating Scale. Mov Disord 27, 1373-1378 (2012).

31. Porras, G., Li, Q. \& Bezard, E. Modeling Parkinson's Disease in Primates: The MPTP Model. Cold Spring Harb Perspect Med 2, a009308 (2012).

32. Meissner, W. G. et al. Priorities in Parkinson's disease research. Nat Rev Drug Discov 10, 377-393 (2011).

33. Kish, S. J. et al. Preferential loss of serotonin markers in caudate versus putamen in Parkinson's disease. Brain 131, 120-131 (2008).

34. Ahmed, M. R. et al. Lentiviral overexpression of GRK6 alleviates L-dopa-induced dyskinesia in experimental Parkinson's disease. Sci Transl Med 2, 28 ra28 (2010).

35. Porras, G. et al. PSD-95 expression controls L-DOPA dyskinesia through dopamine D1 receptor trafficking. J Clin Invest 122, 3977-3989 (2012).

36. Bezard, E. et al. Attenuation of levodopa-induced dyskinesia by normalizing dopamine D3 receptor function. Nat Med 9, 762-767 (2003)

\section{Acknowledgments}

The CNRS and the University Bordeaux 2 (France) provided the infrastructural support.

\section{Author contributions}

M. Morari, E.B. and W.M. designed research; G.P., P.D.D., M. Marti. Q.L., R.M. and R.S performed research; G.P., M. Morari, R.S., E.B. and W.M. analyzed data; G.P., P.D.D., E.B. and W.M. wrote the main manuscript text and W.M. prepared figure 1. All authors reviewed the manuscript.

\section{Additional information}

Financial disclosure E.B. is Chief Scientific Officer of Motac neuroscience Ltd. All other authors reported no biomedical financial interests or potential conflicts of interest.

Competing financial interests: The authors declare no competing financial interests. 
How to cite this article: Porras, G. et al. L-dopa-induced dyskinesia: beyond an excessive dopamine tone in the striatum. Sci. Rep. 4, 3730; DOI:10.1038/srep03730 (2014). (c) (i) $\odot$ This work is licensed under a Creative Commons AttributionBY No NonCommercial-NoDerivs 3.0 Unported license. To view a copy of this license, visit http://creativecommons.org/licenses/by-nc-nd/3.0 